\title{
Médiévales
}

Langues, Textes, Histoire

61 | automne 2011

La chair des émotions

\section{Toucher et être touché : gestes de conciliation et émotions dans les duels judiciaires}

To touch and to be touched: gestures of conciliation and emotions in trial by combat

\section{Klaus Oschema}

\section{OpenEdition}

Journals

Édition électronique

URL : https://journals.openedition.org/medievales/6547

DOI : $10.4000 /$ medievales. 6547

ISSN : 1777-5892

Éditeur

Presses universitaires de Vincennes

Édition imprimée

Date de publication : 20 décembre 2011

Pagination : 142-161

ISBN : 978-2-84292-337-2

ISSN : 0751-2708

\section{Référence électronique}

Klaus Oschema, « Toucher et être touché : gestes de conciliation et émotions dans les duels

judiciaires », Médiévales [En ligne], 61 | automne 2011, mis en ligne le 19 janvier 2012, consulté le 22 avril 2022. URL : http://journals.openedition.org/medievales/6547 ; DOI : https://doi.org/10.4000/ medievales.6547 


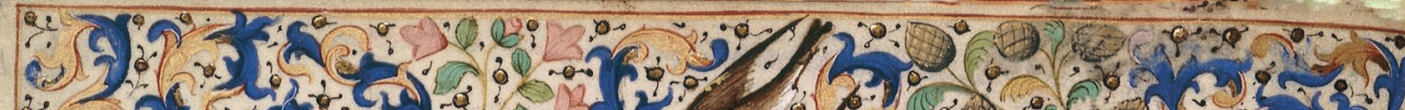

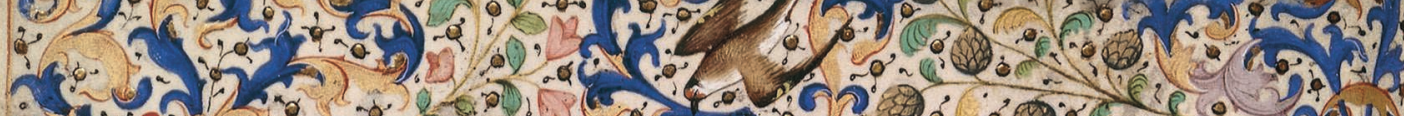
(6)

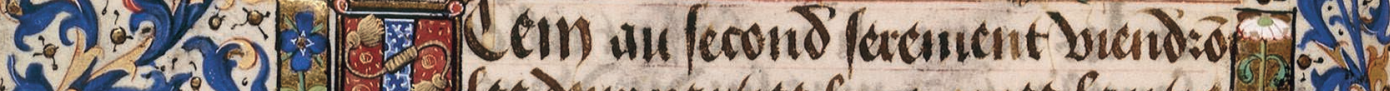
D. पे ก. - $20.6 \%$ -. P. P P.

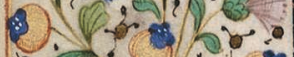
- $a$ 'a.

a (5. )

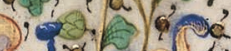

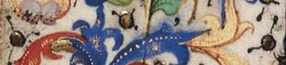

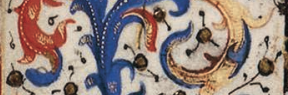

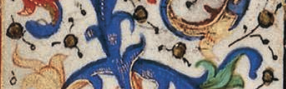
- la car a va

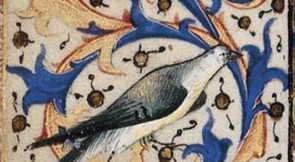
Q2 1 तो ? (riva déy - a ci, G. a 100,8 - ${ }^{2}$ a 90.90 boa a $\bigcup_{-0}$ 3 a. 2 . क. a' $-b_{1}$.

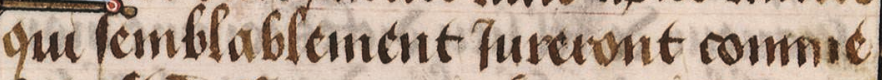

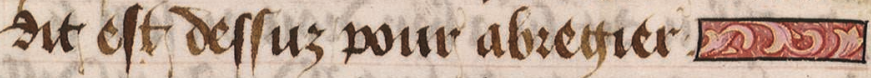

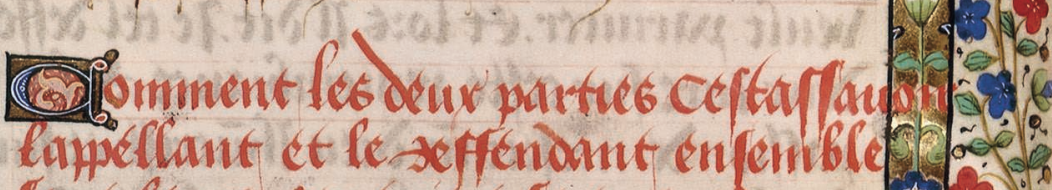

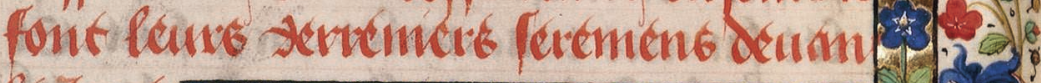

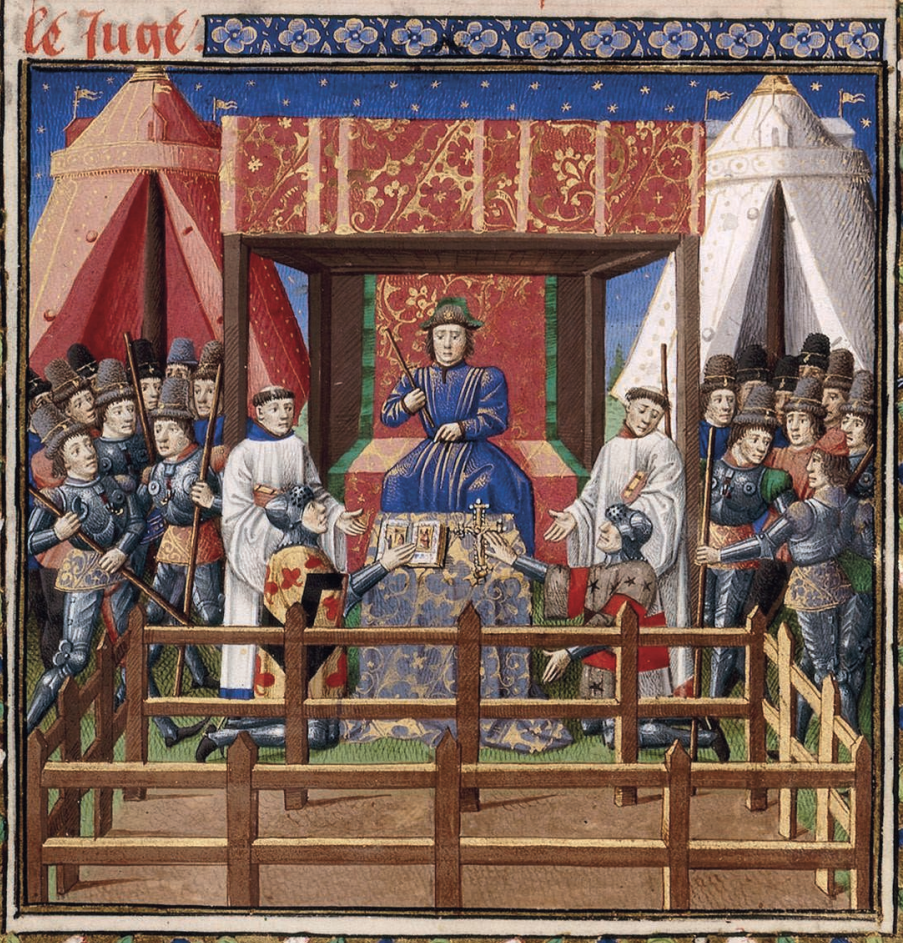
$\operatorname{lom}_{0 \rightarrow}$ a. as

(1)

(.) $\theta^{2}$ 1) ${ }^{2}$. 
Médiévales 61, automne 2011,p. 142-162

\section{Klaus OSCHEMA}

\section{TOUCHER ET ÊTRE TOUCHÉ: GESTES DE CONCILIATION ET ÉMOTIONS DANS LES DUELS JUDICIAIRES}

Au cours des dernières années, le nouvel intérêt pour une histoire des émotions a suscité un grand nombre de débats théoriques et méthodologiques ${ }^{1}$. La présente contribution part de la conviction que la dimension affective de l'existence humaine ne constitue qu'une partie - même si celle-ci est essentielle - des phénomènes que nous devons considérer afin d'élaborer une interprétation adéquate des actions et des acteurs historiques. Afin de montrer comment cette dimension pourrait être intégrée dans l'image que nous nous faisons de l'époque médiévale, elle se concentre sur un détail minuscule qui pourrait paraître anodin dans le cadre général de l'histoire de la France des XIV et Xve siècles.

Or, il me semble que ce détail, qui appartient au domaine des gestes de proximité ${ }^{2}$, permet de montrer les possibilités d'une approche historique qui prend en compte les effets sociaux des émotions. Cette approche peut être appliquée sans que l'historien soit obligé de comprendre la «vraie nature» des émotions en question ou bien leur présence réelle dans une situation donnée. Les émotions des acteurs historiques n'étant accessibles qu'à travers des mécanismes

1. Cf. les titres cités dans les n. 3 et 4 . - Je remercie les organisateurs et les participants du colloque «Le corps des émotions au Moyen Âge» à Aix-en-Provence (23-24 avril 2010) ainsi qu'Andreas Ranft de l'Université de Halle de m'avoir offert l'occasion de présenter et de discuter mes réflexions. Les notes ne prétendant pas être exhaustives, elles indiquent un choix de sources et de travaux dont je me suis servi au cours de la préparation de ce texte. Pour la correction de mes faiblesses linguistiques, je suis très reconnaissant à ma collègue Isabelle Deflers (Heidelberg/Fribourgen-Brisgau), et pour son soutien dans le travail de rédaction.

2. K. Oschema, Freundschaft und Nähe im spätmittelalterlichen Burgund. Studien zum Spannungsfeld von Emotion und Institution, Cologne/Weimar/Vienne, 2006.

Illustration page précédente : Cérémonies des gages de bataille selon les constitutions du bon roi Philippe de France. Le troisième serment des deux adversaires dans un duel judiciaire; les deux parties prêtent serment sur la croix et la Bible, devant le juge diseur.

BnF, ms. fr. 2258 (ms. enluminé, exemplaire du duc François II de Bretagne), f. 18v. 
de transmission et de représentation qui impliquent toute une série d'inflexions ${ }^{3}$, il me semble à la fois faisable et souhaitable d'analyser de manière plus détaillée les stratégies et les mécanismes avec lesquels une société donnée réagit à l'existence des phénomènes affectifs. Ma présentation vise donc moins la «nature» des émotions ${ }^{4}$ et tend plutôt à réfléchir sur la perception de l'affectivité et de ses effets dans une société historique, sur la mise en pratique des positions qui en résultent, ainsi que des possibilités interprétatives qui en découlent pour nos analyses. Bien que les sources sur lesquelles se fondent les réflexions suivantes ne renvoient pas explicitement aux affects, elles permettent de montrer la richesse d'une approche qui inclut l'émotion en tant que catégorie analytique.

L'objet dont il sera ici question fait partie d'un rituel juridique en voie d'extinction au cours du bas Moyen Âge français : il s'agit du duel judiciaire - une pratique dont l'histoire, avec sa lente disparition au cours des $\mathrm{XV}^{\mathrm{e}}$ et $\mathrm{XVI}$ e siècles, a depuis longtemps attiré l'intérêt des historiens ${ }^{5}$. Les approches classiques furent

3. Cf. récemment B. H. Rosenwein, «Problems and Methods in the History of Emotions», Passions in Context [revue en ligne], vol. 1, 2010, http://www.passionsincontext.de/uploads/ media/01_Rosenwein.pdf (26/07/2010),p. 11, n. 37.

4. Sur les débats théoriques récents, voir B. H. Rosenwein, «Worrying about Emotions in History (Review Essay)», American Historical Review, 107 (2002), p. 821-845; EAD., «Thinking Historically about Medieval Emotions », History Compass, 8/8 (2010), p. 828-842; EAD., «Problems and Methods... », et J. Deploige, «Studying Emotions. The Medievalist as Human Scientist? », dans E. Lecuppre-Desjardin et A.-L. Van Bruaene éd., Emotions in the Heart of the City (14th-16th century)/ Les Émotions au cour de la ville (XIV'-XVI' siècle), Turnhout, 2005, p. 3-24. Cf. D. Boquet et P. NAGY éd., Le Sujet des émotions au Moyen Âge, Paris, 2009; R. SchnelL, «Historische Emotionsforschung. Eine mediävistische Standortbestimmung », Frühmittelalterliche Studien, 38 (2004), p. 173-276, et ID., «Emotionsdarstellungen im Mittelalter. Probleme und Aspekte der Referentialität », Zeitschrift für deutsche Philologie, 127 (2008), p. 79-102. L'ouvrage d'E.-M. Engelen, Gefühle, Stuttgart, 2007, p. 8-11, résume les différenciations à faire entre Gefühle (sentiments), Emotionen (émotions), Empfindungen (sensations) et Stimmungen (humeurs).

5. Les remarques suivantes ne prétendent pas à présenter un tableau complet du rôle qu'a joué le duel judiciaire dans les systèmes juridiques médiévaux; il s'agit plutôt d'esquisser le contexte global dans lequel se situe le geste qui m'intéresse ici. Sur l'histoire du duel judiciaire, cf. W. SchiLD, «Zweikampf», Lexikon des Mittelalters, vol. 9, Munich, 1998, col. 723-724. L'étude classique reste H. NotTarP, Gottesurteilstudien, Munich, 1956. Voir aussi H. Holzhauer, «Der gerichtliche Zweikampf. Ein Institut des germanischen Rechts in rechtsethnologischer Sicht» [orig. 1986], dans ID., Beiträge zur Rechtsgeschichte, éd. S. C. SAAR et A. Roth, Berlin, 2000, p. 93-111; M. VALE, «Aristocratic Violence: Trial by Battle in the Later Middle Ages», dans R. W. KAEUPER éd., Violence in Medieval Society, Woodbridge, 2000, p. 159-181, et T. Hiltmann et U. Israel, «"Laissez-les aller". Die Herolde und das Ende des Gerichtskampfes in Frankreich», Francia, 34/1 (2007), p. 65-84. Sur les propositions de duels individuels entre princes, qui ont déjà attiré l'intérêt de J. HuzzingA, Homo ludens. Vom Ursprung der Kultur im Spiel, Hambourg, 18 éd., 2001 [orig. 1938, trad. fr. Homo ludens. Essai sur la fonction sociale du jeu, Paris, 22éd., 1988], p. 103-107, et ID., L'Automne du Moyen Âge, Paris, 1995 [orig. 1919], p. 101-106, cf. en dernier lieu R. SCHNEIDER, «Zweikampf von Königen - statt blutiger Kriege? », dans P. Thorau, S. Penth et R. Fuchs éd., Regionen Europas - 
surtout dominées par l'histoire du droit, qui traitait le duel judiciaire comme une pratique «archaïque» du système judiciaire prémoderne, et par l'histoire culturelle dans le sens d'une «histoire des mœurs». L'étude de Robert Bartlett, parue en 1986, représente un renouvellement en ce qu'elle inclut la perspective d'une histoire des mentalités ainsi que d'une anthropologie historique. Sur cette base, Bartlett souligne la différence entre l'ordalie par l'eau et par le feu d'un côté, et le duel judiciaire de l'autre ${ }^{6}$. Les deux premières relevant profondément de la culture chrétienne, elles nous confrontent donc à une histoire bien cernée, qui connaît un début avec la christianisation des peuples germaniques ${ }^{7}$ et la création d'une organisation sociale et judiciaire reposant essentiellement sur la dimension chrétienne, et une fin assez bien identifiable marquée par le concile du Latran IV (1215) lors duquel l'Église officielle a pris ses distances face à ces pratiques ${ }^{8}$.

L'histoire du duel judiciaire, en revanche, suit un développement plus flou: il semble qu'elle remonte à des origines lointaines dans les sociétés «archaïques ${ }^{9}$, et la disparition du duel en tant que pratique sanctionnée par les systèmes judiciaires ne résulte pas d'une politique explicite. Nous avons plutôt

Europa der Regionen. Festschrift für Kurt-Ulrich Jäschke zum 65. Geburtstag, Cologne/Weimar/ Vienne, 2003, p. 21-32, et L. Vones, «Un mode de résolution des conflits au bas Moyen Âge: le duel des princes », dans P. Contamine et O. GuyotJEAnNin éd., La Guerre, la violence et les gens au Moyen Âge. Vol. I: Guerre et violence, Paris, 1996, p. 321-332. Sur le développement ultérieur du duel moderne, cf. P. Brioist, H. Drévillon et P. SERna, Croiser le fer. Violence et culture de l'épée dans la France moderne (XVI'-XVIII siècle), Seyssel, 2002; U. FreVERT, Ehrenmänner. Das Duell in der bürgerlichen Gesellschaft, Munich, 1995; F. BiLlacois, Le Duel dans la société française des XVI -XVII siècles. Essai de psychosociologie historique, Paris, 1986. De caractère populaire: M. Monestier, Duels. Histoire, techniques et bizarreries du combat singulier, des origines à nos jours, Paris, 2005, ici p. 69-95. Ne m'était pas encore accessible S. Neumann, Der gerichtliche Zweikampf: Gottesurteil - Wettstreit - Ehrensache, Ostfildern, 2010; à l'Université de Bamberg, Anika Auer prépare actuellement une thèse sur Gottesurteile im Kontext rechtlich-politischer und kultureller Wandlungsprozesse im europäischen Mittelalter.

6. R. BARTLET, Trial by Fire and Water. The Medieval Judicial Ordeal, Oxford, 1986; cf. E. CoHen, The Crossroads of Justice. Law and Culture in Late Medieval France, Leyde/New York/ Cologne, 1993, p. 56-62.

7. Un sujet qui a suscité beaucoup d'intérêt au cours des dernières années, cf. B. DumÉzIL, Les Racines chrétiennes de l'Europe. Conversion et liberté dans les royaumes barbares (Ve-VIII siècle), Paris, 2005, et D. KöNIG, Bekehrungsmotive. Untersuchungen zum Christianisierungsprozess im römischen Westreich und seinen romanisch-germanischen Nachfolgern (4.-8. Jh.), Husum, 2008.

8. R. BARTLETT, Trial..., p. 98-100.

9. Ibid., p. 103-105; cf. H. Holzhauer, «Der gerichtliche Zweikampf...», p. 102, qui souligne la spécificité «européenne» ou «indo-européenne» de la pratique; celle-ci ne constitue donc pas un élément universel des sociétés soi-disant «primitives» (ibid., p. 95 sq.). Voir aussi H. NotTarp, Gottesurteilstudien, p. 269-313. Je rappelle que le niveau de complexité de l'organisation sociale d'une culture donnée est largement indépendant de sa disposition envers la force physique et l'agressivité, cf. R. WALZ, «Strategien der Gewaltvermeidung. Ein Vergleich segmentärer mit bäuerlichen Gesellschaften in Europa», dans S. EsDERS éd., Rechtsverständnis und 
affaire à une tendance générale des pouvoirs centraux à imposer leur domination, leurs droits seigneuriaux et leur compétence juridique à travers des mécanismes judiciaires qui leur permettaient de trancher les questions et les litiges qui se développaient sur leurs territoires. Admettre une procédure qui remettait la prise de décision entre les mains d'une instance quasiment «insaisissable» équivalait alors à une perte de pouvoir ${ }^{10}$.

Bien que nous puissions donc identifier des motivations et des intérêts ayant déterminé les souverains à mener une politique visant à abolir la pratique des duels judiciaires, il est plus difficile de saisir une stratégie politique consciente - si l'on fait abstraction de l'interdiction officielle de la part de l'Église à partir de 1215 et de certaines tentatives des rois de France d'imposer cette interdiction dans leur pays. Sommairement on peut dire que le développement résulta plutôt des lentes mutations et des changements structurels qui ne concernaient pas seulement la dimension «gouvernementale», mais également les attitudes sociales et mentales au sens large. Qu'on ne pense qu'à l'adoption progressive du droit civil romain, qui favorisait de nouvelles procédures afin de trancher des questions juridiques. Au niveau pratique, l'ensemble de ces influences se manifestait dans le royaume de France sous forme d'une interdiction formelle des duels judiciaires, décrétée par le roi Philippe IV en $1304^{11}$.

Malgré cette interdiction, qui ne faisait qu'appliquer les exigences de la papauté ${ }^{12}$, la pratique du duel continuait à exister quoique de manière quelque peu marginalisée. C'est la juridiction royale elle-même qui nous en fournit la preuve car deux ans après l'interdiction de 1304, Philippe IV revenait sur sa

Konfliktbewältigung. Gerichtliche und außergerichtliche Strategien im Mittelalter, Cologne-WeimarVienne, 2007, p. 211-238.

10. Cf. la disparition progressive de l'institution de l'arbitrage: bien qu'elle ait subsisté sous différentes formes jusqu'à l'époque moderne, elle fut appliquée moins régulièrement vers la fin de l'époque médiévale. Sur la tendance à remplacer l'arbitrage par l'autorité juridique du seigneur (qui pouvait ensuite gracier les personnes condamnées), cf.l'exemple savoyard: N. CARRIER, «Une justice pour rétablir la "concorde": la justice de composition dans la Savoie de la fin du Moyen Âge (fin $\mathrm{XIII}^{\mathrm{e}}$-début $\mathrm{XVI}^{\mathrm{e}}$ siècle)», dans Le Règlement des conflits au Moyen Âge ( $\mathrm{xxxI}^{\mathrm{e}}$ Congrès de la SHMES Angers, juin 2000), Paris, 2001, p. 237-257. Voir aussi C. GAuvARD, «Pouvoir de l'État et justice en France à la fin du Moyen Âge», dans J.-P. GenEt éd., Rome et l'État moderne européen, Rome, 2007, p. 341-364.

11. Texte dans Ordonnances des rois de France de la troisième race, 22 vol., vol. I, Paris, 1732-1847, p. 390-392. La tentative d'interdiction des duels judiciaires de Louis IX provoqua la protestation des nobles, cf. T. Hiltmann et U. IsRaEL, «"Laissez-les aller”...», p. 76.

12. À côté de l'interdiction promulguée par le concile de Latran IV en 1215, différents papes veillaient de manière active sur son application, cf. par exemple Les Registres d'Innocent IV, publiés ou analysés d'après les manuscrits originaux du Vatican et de la Bibliothèque nationale, éd. É. Berger, vol. III, Paris, 1897, nº 5883, p. 92 sq., et n 6184, p. 148 sq. (1252); voir déjà H. NOTTARP, Gottesurteilstudien, p. 364. Sur l'interdiction et les réactions des princes séculiers, cf. R. BARTLETT, Trial... (n. 6), p. 118-120. 
décision. En 1306, il publia une ordonnance qui réglait les conditions dans lesquelles un tel combat devait encore être permis: l'existence d'un crime devait être manifeste; ce crime devait être un crime capital qui méritait d'être puni de mort; la vérité ne pouvait être établie par aucun autre moyen de preuve; l'accusé devait être suspect ${ }^{13}$. Par la suite, c'était surtout dans le monde de la noblesse, qui se définissait toujours par le privilège de défendre son droit et son honneur au moyen des armes ${ }^{14}$, que le duel jouait un rôle important et fournissait matière à des traités théoriques consacrés à son organisation et à son déroulement de manière correcte ${ }^{15}$. Si ces traités furent habituellement rédigés par des auteurs nobles - entre autres le célèbre Olivier de La Marche ${ }^{16}$-, des prescriptions détaillées nous sont également parvenues de la part de spécialistes du système judiciaire, tel l'avocat Guillaume Du Breuil, l'auteur du Stilus curie parlamenti ${ }^{17}$. Tous ces éléments nous permettent de reconstruire de manière concrète et détaillée le déroulement d'un duel juridique ${ }^{18}$, comme le cas célèbre qui marque à la fois l'approche de la fin de cette tradition et qui a récemment été analysé par l'historien américain Eric Jager. Il s'agit du duel qui a confronté en 1386 le chevalier Jean de Carrouges et l'écuyer Jacques Le Gris, deux nobles normands dont le litige n'a pas pu être tranché d'une autre manière ${ }^{19}$. En se fondant sur

13. Le texte de l'ordonnance est édité dans Ordonnances des rois de France..., vol. I, p. 435-441; cf. T. Hiltmann et U. IsRaEL, «"Laissez-les aller”...», p. 76, et surtout T. Hittmann, Spätmittelalterliche Heroldskompendien. Referenzen adeliger Wissenskultur in Zeiten gesellschaftlichen Wandels (Frankreich und Burgund, 15. Jahrhundert), Munich, 2011, p. 300-331. Pour les accusations qui pouvaient mener à un gage de bataille, cf. ibid., p. 300, et R. BARTLETT, Trial..., p. 106-109.

14. Cf. M. Keen, Chivalry, Londres, 1984, p. 151-153; R. W. Kaeuper, War, Justice, and Public Order. England and France in the Later Middle Ages, Oxford, 1988; M. VALE, War and Chivalry: Warfare and Aristocratic Culture in England, France and Burgundy at the End of the Middle Ages, Londres, 1981, et surtout P. Contamine, La Noblesse au royaume de France de Philippe le Bel à Louis XII. Essai de synthèse, Paris, 1998.

15. Le recul du nombre des gages de bataille a été observé pour le Parlement de Paris par C. Gauvard, De grace especial. Crimes, État et société en France à la fin du Moyen Âge, 2 vol., vol. I, Paris, 1991, p. 172-179.

16. Olivier de La Marche, «Livre de l'advis de gaige de bataille», dans B. Prost éd., Traités du duel judiciaire, relations de pas d'armes et tournois, Paris, 1872, p. 1-54 (inclut le «Livre du seigneur de l'Isle-Adam pour gaige de bataille», ibid., p. 28-41).

17. Guillaume Du Breull, Stilus curie parlamenti, éd. F. Aubert, Paris, 1909, ici p. 101-123.

18. Malgré le nombre de renvois historiographiques qui ne mentionnent que le fait et le résultat des duels, cf. par ex. Enguerrand de Monstrelet, Chronique, éd. L. DouËt-D'ArcQ, vol. 1, Paris, 1858, p. 99 sq. (duel entre les «gentilshommes» Bornete et Sohier Barnage au Quesnoy en 1405); cf. T. Hiltmann et U. Israel, «"Laissez-les aller"...», p. 77.

19. E. JAGER, Auf Ehre und Tod. Ein ritterlicher Zweikampf um das Leben einer Frau, Munich, 2006 [orig. américain 2004; trad. fr. Le Dernier Duel. Paris, 29 décembre 1386; pour des raisons pratiques j'utilise la version allemande]. Le duel de 1386 est fréquemment interprété comme le «dernier combat juridique autorisé par le Parlement de Paris», cf. ibid., p. 229; voir cependant T. Hiltmann et U. IsRael, «"Laissez-les aller"... », p. 65. Le récit le plus célèbre en est celui de JEAN 
l'accusation de sa femme, Jean de Carrouges jurait que, profitant de son absence, son adversaire avait abusé de son épouse et l'avait violée, tandis que l'accusé se déclarait innocent.

Je n'entre pas ici dans les détails de ce cas qui semble insoluble à tout jamais ${ }^{20}$. Ce qui m'intéresse ici, c'est surtout le déroulement ritualisé du combat qui suivit les réglementations établies par Philippe le Bel et précisées ensuite, car même si le duel juridique est aujourd'hui perçu comme une procédure archaïque ou «primitive», son déroulement suivait en réalité un plan précis qui réglait pratiquement tous les actes des participants et du public - de la préparation des combattants, en passant par leur arrivée sur le champ de combat et leur présentation au juge, jusqu'à l'ouverture du combat proprement dit ${ }^{21}$.

Dans le déroulement de ces événements, un moment du rituel est codifié de manière particulièrement précise - et cela m'amène finalement au cœur du sujet: il s'agit des serments que les adversaires prêtent en public afin de souligner la légitimité de leur cas. Après l'entrée dans les lices et la présentation - d'abord du plaignant (l'appellant), ensuite de l'accusé (le deffendant) -, qui est rythmée par trois cris du héraut concernant le comportement du public ${ }^{22}$, l'appelant prête un premier serment, confirmant qu'il a

certainnement bonne, juste et saincte querelle et bon droit d'avoir en ce présent gaige de bataille appellé [le tel, comme faulx et mauvaiz traytre, ou murtrier, ou foy mentie, selon le cas qu'il est; et lequel a très faulse et très mauvaise cause et querelle de soy en deffendre]. Et ce je lui monstreray aujourd'hui par mon corps contre le sien, à l'ayde de Dieu, de Nostre Dame, et de monseigneur Saint George le bon chevalier ${ }^{23}$.

Le texte des Cérémonies des gages de bataille, qui élabore considérablement sur l'ordonnance de $1306^{24}$, prévoit que ce serment doit être prononcé en présence d'un prêtre et d'un maréchal, et que l'appelant doit prononcer les paroles en

Froissart, CEuvres, éd. J.-B.-M.-C. Kervyn de Lettenhove, vol. XII, Bruxelles, 1871, p. 29-39 et 366-373 (récits et documents supplémentaires).

20. Cf. pourtant la présentation de E. JAGER, Auf Ehre und Tod..., p. 237-245.

21. Ibid., p. 168-214; T. Hiltmann et U. IsRael, «"Laissez-les aller”...», p. $76-79$ et 83 sq., se concentrent sur le rôle des hérauts.

22. Cf. Guillaume Du Breuil, Stilus curie..., p. 111-115; Cérémonies des gages de bataille selon les constitutions du bon roi Philippe de France, éd. G.-A. Crapelet, Paris, 1830, p. 12-22; Olivier De La Marche, «Livre du seigneur de l'Isle-Adam», p. 31-36.

23. Cérémonies des gages de bataille..., p. 24.

24. Voir T. Hiltmann, Spätmittelalterliche Heroldskompendien..., p. 307-320, qui distingue trois versions. Le texte des Cérémonies réunit l'ordonnance originelle, des commentaires et la description des cérémonies qui datent probablement du milieu du XIV e siècle, cf. ibid., p. 302-307. L'ensemble fut intégré dans les coutumes bourguignonnes, cf. Le Coutumier bourguignon glosé (fin $d u$ XIv siècle), éd. M. Petitjean, M.-L. Marchand et J. Metman, Paris, 1982, p. 299-311. Pour les 
touchant une croix avec sa main droite et un missel avec sa main gauche sans enlever ses gants ${ }^{25}$. Ce contact indirect entre celui qui prête serment et l'objet sacré, qui sert à confirmer la validité de la parole, se répète lors du premier serment de l'accusé et lors du deuxième serment des deux parties, qui concerne avant tout les détails du combat car les deux adversaires jurent de n'employer que des moyens honnêtes ${ }^{26}$.

Ce n'est donc qu'au cours du troisième serment, prêté par les deux adversaires ensemble en même temps, que le rituel prévoit un contact physique direct. Pour la première fois, les deux acteurs se touchent sans que leur contact soit affaibli par l'emploi d'un objet qui créerait une barrière - une différence fondamentale en comparaison avec le deuxième serment, qui a été prêté les mains gantées. En outre, à la place des serments individuels, les combattants se présentent maintenant ensemble devant leur juge et s'agenouillent en face de la croix et du missel (voir illustration p. 142). En accord avec le texte, l'enlumineur de l'exemplaire des Cérémonies du duc François II de Bretagne insiste sur le fait que les mains des protagonistes doivent être nues, mais il ne met pas en scène le contact physique entre les adversaires. Or, le rituel prévoit explicitement que le maréchal leur fera enlever leurs gants, qu'il mettra ensuite sur les bras de la croix $^{27}$. Afin de souligner le caractère sacré de l'action qui suit, celle-ci doit être exécutée en présence d'un prêtre qui rappelle aux acteurs la souffrance de JésusChrist et la punition sévère qui attend le parjure ${ }^{28}$. Ensuite les jureurs prononcent les paroles que leur dicte le maréchal. L'ordonnance souligne encore une fois l'arrangement spatial et gestuel lors du serment qui doit être prononcé «quant ils s'entreverront et s'entretiendront par les mains ${ }^{29} »$.

variantes qui ont circulé en Angleterre, voir The Black Book of the Admiralty, éd. T. Twiss, vol. I, Londres, 1871, p. 300-344.

25. Cérémonies des gages de bataille..., p. 23 ; cf. Olivier de La MARCHE, «Livre du seigneur de l'Isle-Adam», p. 36. Le Stilus curie ne connaît qu'un seul serment (qui équivaut donc au dernier des trois serments) qui inclut un contact physique: celui qui prête serment touche son adversaire avec la main gauche et tient sa main droite au-dessus d'un livre d'évangiles, cf. Guillaume Du BREUIL, Stilus curie..., p. 115 sq. ( «... arripiet adversarium suum cum manu sinistra, et dexteram tenebit super sancta [evangelia], et dicet verba que sequuntur:...».) Ici la présence (passive) d'un prêtre est prévue. Sur les différences entre le Stilus curie et les autres versions des Cérémonies, cf. T. Hiltmann, Spätmittelalterliche Heroldskompendien... p. 307-309.

26. Olivier de La Marche, «Livre du seigneur de l'Isle-Adam», p. 36 sq. Le texte des Cérémonies des gages de bataille..., p. 26, abrège considérablement.

27. Cérémonies des gages de bataille..., p. 27 ; Olivier DE LA MARCHE, «Livre du seigneur de l'Isle-Adam», p. 36 sq.

28. Cérémonies des gages de bataille..., p. 27; il s'agit du seul moment qui prévoit une action précise d'un clerc, cf. R. BARTLETt, Trial..., p. 121.

29. Cérémonies des gages de bataille..., p. 28. 
Les paroles du serment ne contiennent rien de surprenant car elles ne font que réitérer le contenu des deux premiers ${ }^{30}$. Or, cette dernière étape avant le combat proprement dit est tout de même caractérisée par une nouvelle qualité au niveau de l'encadrement (avec l'intervention du prêtre), mais surtout en ce qui concerne l'intensification du domaine gestuel; lors du troisième serment, les deux adversaires sont confrontés l'un à l'autre de manière immédiate pour la première fois au cours du rituel. Le contact physique avec les objets sacraux devient également plus intense car au lieu de les toucher avec leurs mains, les jureurs embrassent maintenant et la croix et le livre des Évangiles ${ }^{31}$.

Ce contact entre l'objet sacré et la bouche des acteurs accentue encore la dimension religieuse et donc l'autorité divine à laquelle le serment se réfère en dernier lieu ${ }^{32}$. Si la signification de ce geste semble bien accessible, l'autre détail du rituel ne peut qu'étonner, car comme nous l'avons vu, les Cérémonies prévoient explicitement que ce dernier serment doit être prononcé pendant que les deux acteurs se tiennent par la main. En fait, le texte insiste encore une fois sur ce geste de proximité physique: après l'accomplissement du serment solennel, le maréchal qui veille sur la suite des événements prend les mains des adversaires et les réunit ${ }^{33}$. Dans cette position, caractérisée par le contact immédiat, les deux parties répètent une ultime fois leurs accusations mutuelles, tout en soulignant réciproquement la position défectueuse de l'adversaire:

$\mathrm{O}$ tu, tel, que je tiens par la main droite, par les seremens que j'ay fais, la cause dont je t'ay appellé est vraye, par quoy j'ay bonne cause et loyale de toy appeller, et à ce jour t'en combatray. Et tu as mauvaise cause et nulle querelle de t'en combatre et deffendre contre moy, et tu le scez, dont j'en appelle Dieu et monseigneur Saint George le bon chevalier à tesmoing, comme faulx, traytre et foy mentie que tu es ${ }^{34}$.

30. Ibid., p. 28 sq.

31. Ibid., p. 29: «Et sur ce je baise ceste vraye croix et les sainctes Évangiles, et me tais.»

32. Sur la dimension symbolique du baiser, cf. K. Oschema, Freundschaft ..., p. 488-538, avec des renvois bibliographiques.

33. Cérémonies des gages de bataille..., p. 29: «[...] le mareschal les prent par les deux mains droites, et les fait entretenir.» OLIVIER DE LA MARCHE, «Livre du seigneur de l'Isle-Adam», p. 37, donne une version simplifiée qui résume le serment entier sous la formule qui suit, cf. n. 34 .

34. Cérémonies des gages de bataille..., p. 29; pour le serment de l'accusé, voir ibid., p. 30. Cf. le commentaire anonyme du milieu du xve siècle au Plaict-général du 3 mai 1368 de Lausanne, prévoyant que les protagonistes se tiennent par la main gauche: «[...] dicti pugiles debent se tenere et tangere manus suas sinistras utriusque modi suasque manus debent habere supra corpus Christi, dicendo sic: [...] ego talis appelans iuro per sacrum corpus Christi quod habui iustam et rationalem causam appellandi de duello talem (nominetur) quem teneo manum et proposita per me in appellatione contra dictum talem (nominetur) sunt vera [...].»Voir Les Sources du droit du canton de Vaud. Moyen Âge $\left(X^{e}-X V I^{e}\right.$ siècle $)$. B. Droits seigneuriaux et franchises municipales. I. Lausanne et les terres épiscopales, éd. D. AneX-Cabanis et J.-F. Poudret, Aarau, 1977, nº 190, p. 239-349 (ici 331 sq.). 
Après ces dernières paroles, chacun des deux embrasse encore une fois la croix, avant de se lever simultanément avec son adversaire pour rentrer dans son pavillon où il attend le début du combat ${ }^{35}$.

À première vue, il est vrai, cette suite d'événements ne pose pas beaucoup de problèmes d'interprétation : nous avons affaire à un rituel classique, le serment, qui jouait un rôle bien établi dans le monde du droit depuis les premiers siècles du Moyen Âge ${ }^{36}$. Dans sa version juridique, cette « condamnation conditionnelle de soi-même» (bedingte Selbstverfluchung) ${ }^{37}$ était depuis longtemps acceptée comme moyen de preuve sous la forme d'un serment purgatoire (Reinigungseid) qui établissait l'innocence de l'accusé ${ }^{38}$. Or, dans le cas d'un gage de bataille le serment ne tranchait pas la question de l'accusation mais il servait bien au contraire à confirmer la légitimité de chacune des parties et à soutenir les accusations mutuelles. Par conséquent, il est évident qu'un des deux acteurs devait forcément être parjure, ce qui explique la position défavorable de l'Église envers ce genre de procédure.

Quant aux éléments rituels et gestuels que nous pouvons observer dans les textes normatifs et dans certaines descriptions de duels judiciaires, ils ne semblent pas surprenants non plus: toucher un objet sacral pendant la prononciation d'un serment faisait partie des actes habituels de l'époque ${ }^{39}$. Même le contact

Sur la datation du commentaire et l'attribution à Jean de May, voir ibid., p. 215. Cf. H. NotTARP, Gottesurteilstudien..., p. 277.

35. Cérémonies des gages de bataille..., p. 30. Ce déroulement est confirmé par OLIVIER DE LA MARCHE, «Livre du seigneur de l'Isle-Adam», p. 36-38, qui mentionne cependant la main gauche!

36. Cf. H. BeCK et G. Köвler, «Eid», Reallexikon der germanischen Altertumskunde, vol. VI, $2^{\mathrm{e}}$ éd., Berlin-New York 1986, p. 537-542, et D. Munzel-Everling, «Eid», Handwörterbuch zur deutschen Rechtsgeschichte, vol. I, $2^{\mathrm{e}}$ éd., Berlin, 2004, col. 1249-1261. Pour les différents contextes dans lesquels le serment jouait un rôle juridique, voir récemment les études exemplaires réunies dans S. EsDERs éd., Rechtsverständnis und Konfliktbewältigung...

37. Cf. H. Beck et G. Köbler, «Eid», p. 40 ; voir aussi A. Holenstein, «Rituale der Vergewisserung: Der Eid als Mittel der Wahrheitsfindung und Erwartungsstabilisierung im Mittelalter und in der frühen Neuzeit», dans E. Bierende, S. Bretfeld et K. Oschema éd., Riten, Gesten, Zeremonien. Gesellschaftliche Symbolik in Mittelalter und früher Neuzeit, Berlin-New York, 2008, p. 229-250 (ici 233 sq.), et les contributions dans P. Friedrich et M. SchNeIDER éd., Fatale Sprachen: Eid und Fluch in Literatur- und Rechtsgeschichte, Paderborn-Munich, 2009, dont les raisonnements sont inspirés par G. Agamben, Le Sacrement du langage. Archéologie du serment (Homo sacer II, 3), trad. J. Gayraud, Paris, 2009.

38. Sur la dynamique du doute et du soupçon que pouvait entraîner un serment purgatoire, cf. M. NieHAus, «Tortura spiritualis. Schwören unter Beobachtung », dans P. FrIEdRICH et M. SCHNEIDER éd., Fatale Sprachen..., p. 121-137 (ici 128-130). H. NotTARP, Gottesurteilstudien (n. 5), p. 270, interprète la sommation à un duel judiciaire comme une opposition préemptive au serment purgatoire de la part de l'accusateur.

39. L. Kolmer, Promissorische Eide im Mittelalter, Kallmünz, 1989; R. Schmidt-Wiegand, «Eid und Gelöbnis, Formel und Formular im mittelalterlichen Recht», dans P. ClassEN éd., Recht 
physique entre les acteurs ne constituait pas un élément extraordinaire ${ }^{40}$ : il suffit de penser ici au rituel féodal ${ }^{41}$. Grâce aux travaux de Jacques Le Goff et d'autres, nous connaissons bien aujourd'hui la gamme et le déroulement des gestes qui accompagnaient l'acte d'hommage dans sa version classique: l'agenouillement du vassal devant son seigneur, l'immixtio manuиm et le baiser qui rétablissait une égalité symbolique en exprimant le caractère réciproque de la relation personnelle ${ }^{42}$.

Or, malgré le caractère peu extraordinaire des catégories de gestes exécutés dans notre contexte, une analyse plus détaillée révèle des aspects qui restent problématiques si on limite l'interprétation au domaine juridique proprement $\mathrm{dit}^{43}$. Ceci devient plus clair si on se concentre sur la signification du contact physique entre les acteurs: si les attouchements ne sont aucunement inconnus, ni dans le contexte juridique, ni dans la culture chevaleresque et nobiliaire (où on demandait aux adversaires de « [se] toucher ensemble ${ }^{44} »$ à la fin d'un combat lors d'un tournoi), le contact qui précède le combat juridique reste curieux. Car toucher exprimait avant tout un lien ${ }^{45}$ - avec une autre personne ou un objet et le contact immédiat était caractérisé, dans la perception des contemporains, par une efficacité qui transcendait la dimension purement corporelle ${ }^{46}$. Or, quel

und Schrift im Mittelalter, Sigmaringen, 1977, p. 55-90. Cf. sur la pratique de Louis XI de jurer sur la vraye croix: J.-M. MATZ, «Religion et politique à la fin du Moyen Âge: la vraie croix de SaintLaud d'Angers», Annales de Bretagne et des Pays de l'Ouest, 94 (1987), p. 241-263 (ici 249-253).

40. Dans le contexte du serment, cf. F. Billacois, «Le corps jureur: pour une phénoménologie historique des gestes du serment», dans R. Verdier éd., Le Serment, vol. I, Paris, 1991, p. 93-101.

41. Voir l'article pionnier de J. Le Goff, «Le rituel symbolique de la vassalité», dans ID., Pour un autre Moyen Âge. Temps, travail et culture en Occident: 18 essais, Paris, 1977, p. 349-420.

42. K. Oschema, Freundschaft..., p. 500-508; K. Van Eickels, Vom inszenierten Konsens zum systematisierten Konflikt. Die englisch-französischen Beziehungen und ihre Wahrnehmung an der Wende vom Hoch-zum Spätmittelalter, Stuttgart, 2002, p. 312-333, et ID., «Kuß und Kinngriff, Umarmung und verschränkte Hände. Zeichen personaler Bindung und ihre Funktion in der symbolischen Kommunikation des Mittelalters», dans J. Martschukat et S. Patzold éd., Geschichtswissenschaft und "Performative Turn». Ritual, Inszenierung und Performanz vom Mittelalter bis zur Neuzeit, Cologne-Weimar-Vienne, 2003, p. 133-159.

43. Cf. R. Schmidt-WIEGAND, «Gebärden», Handwörterbuch zur deutschen Rechtsgeschichte, vol. I, $2^{\mathrm{e}}$ éd., 2004, col. 1954-1969; EAD., «Gebärdensprache im mittelalterlichen Recht», Frühmittelalterliche Studien, 16 (1982), p. 363-379.

44. Voir K. Oschema, Freundschaft..., p. 336-340, pour plus d'exemples.

45. Ainsi J. A. Burrow, Gestures and Looks in Medieval Narrative, Cambridge, 2002, p. 32-38 (poignée de main) et p. 11-16 (tenir la main).

46. Qu'on ne pense qu'à l'attouchement guérissant des «rois thaumaturges», cf. M. BLOCH, Les Rois thaumaturges. Étude sur le caractère surnaturel attribué à la puissance royale particulièrement en France et en Angleterre, Paris, 1924, et J. EHLERS, «Der wundertätige König in der monarchischen Theorie des Früh- und Hochmittelalters », dans P.-J. HeINIG et al.éd., Reich, Regionen und Europa in Mittelalter und Neuzeit. Festschrift für Peter Moraw, Berlin, 2000, p. 3-19, ainsi qu'à l'interdiction de toucher un roi sacré, cf. K. Oschema, Freundschaft..., p. 393-398. 
rôle attribuer à une telle action dans le contexte d'un gage de bataille ${ }^{47}$ ? Afin de sanctionner la parole de l'accusation ou bien celle de la défense, on aurait pu se satisfaire de la prononciation réitérée de ces paroles en présence d'un objet sacral - la croix et l'Évangile ${ }^{48}$. Même l'identification en public de l'adversaire respectif ne nécessitait pas le contact physique, mais un simple geste indicatif aurait pu suffire ${ }^{49}$.

Certes, les détails du contact physique mentionnés dans nos sources varient considérablement et il semblerait qu' un geste de la main gauche aurait pu signifier, aux yeux du public, une «disharmonie», comme l'ont constaté plusieurs interprètes modernes du rituel du gage de bataille ${ }^{50}$. Les liens positifs, comme celui de la vassalité, du mariage, du contrat et de la paix, étaient habituellement exprimés par les partenaires qui se donnaient la main droite ${ }^{51}$. Or, l'imaginaire de l'inimitié et du conflit reposait avant tout sur la mise en scène de la distance on évitait tout contact physique avec l'adversaire ainsi que certaines paroles (la salutation verbale ou gestuelle) - afin d'exprimer la confrontation imminente ${ }^{52}$. En outre, nos sources sont loin d'être d'accord sur la question de savoir quelle

47. Le geste accompli selon le cérémoniel français se distingue bien des gestes juridiques dans la sphère germanique où l'accusateur traîne l'accusé devant le juge en le saisissant au col, cf. H. NotTARP, Gottesurteilstudien..., p. 273.

48. A. Holenstein, «Rituale der Vergewisserung...», p. 235-242.

49. Sur la relation étroite entre «indiquer» et «accuser» dans la rhétorique classique, cf. les brèves remarques de H. PomPE, «Eine Handreichung zum Thema», dans M. BicKENBACH, A. KLAPPERT et H. Pompe éd., Manus Loquens. Medium der Geste - Gesten der Medien, Cologne, 2003, p. 8-25 (ici 15). Au-delà de l'histoire de l'art (cf. A. GAMPP, «Antikendämmerung. Vom eingeschränkten Gebrauch der Gesten als Bildsprache im frühen Mittelalter», dans E. Bierende, S. BRETFELD et K. Oschema, Riten, Gesten..., p. 3-20 (ici 19), et J. C. Griffin, Pointing Gestures in Medieval Miniatures. A Study Based on Illustrated Manuscripts of the Terence Comedies, Ann Arbor, 1993), les gestes déictiques n'ont pas beaucoup retenu l'attention des médiévistes. Cf. H. Wenzel et C. S. JAEGER éd., Visualisierungsstrategien in mittelalterlichen Bildern und Texten, Berlin, 2006, et l'ouvrage de référence de J.-C. Sснмітт, La Raison des gestes dans l'Occident médiéval, Paris, 1990. Pour une approche sémiotique, cf. E. FrICKE, Origo, Geste und Raum. Lokaldeixis im Deutschen, Berlin, 2007, et A. Kendon, Gesture. Visible Action as Utterance, $2^{\mathrm{e}}$ éd., Cambridge, 2005, p. 199224 (ch. 11: «On Pointing»).

50. E. JAGER, Auf Ehre und Tod..., p. 189.

51. Sur les gestes de la main en France et en Bourgogne à la fin du Moyen Âge, cf. K. Oschema, Freundschaft..., p. 398-474; sur le domaine anglais, J. A. Burrow, Gestures..., p. 11-16, 32-38 et passim. La dichotomie «gauche»/«droite» est au centre de l'étude pionnière de R. HERTZ, «La prééminence de la main droite. Étude sur la polarité religieuse», Revue philosophique, 68 (1909), p. 553-580.

52. K. Oschema, Freundschaft..., p. 334 sq.; H. Fuhrmann, «"Willkommen und Abschied". Über Begrüßungs- und Abschiedsrituale im Mittelalter», dans W. HaRtmann éd., Mittelalter. Annäherungen an eine fremde Zeit, Ratisbonne, 1993, p. 111-139 (ici 120 sq.) 
main devait être utilisée dans le rituel ${ }^{53}$. Pourquoi donc les adversaires d'un duel judiciaire devaient-ils se toucher?

Afin de résoudre cette énigme, la prise en compte des phénomènes affectifs et des stratégies contemporaines mises en œuvre pour les moduler peut ouvrir de nouvelles pistes. Car si nous faisons abstraction du contexte de la confrontation, les adversaires des gages de bataille ne font rien d'autre qu'exécuter des gestes de conciliation ${ }^{54}$. Bien que ces actions se déroulent dans un cadre rituel contraignant, il n'est pas exclu de les interpréter comme une dernière tentative afin d'éviter l'éclat de violence qui devait suivre. Si cette idée semble peu probable au premier abord ${ }^{55}$, l'attitude générale de la culture de l'époque envers les mécanismes de l'affectivité et le façonnement des relations personnelles qui en découle, permet de retenir une telle interprétation.

Ainsi, en parcourant quelques textes historiographiques des XIV et $\mathrm{XV}^{\mathrm{e}}$ siècles, on découvre que les auteurs renvoient plus d'une fois à la force mystérieuse qu'exerce le contact immédiat entre deux personnes antagonistes. Ne citons ici que l'exemple du conflit entre Hugues d'Arpajon et Amaury de Sévérac, un épisode des luttes internes qui déchiraient la maison d'Armagnac dans les années 1420. Selon la Chronique de la Pucelle, un texte bien postérieur, ce conflit n'a longtemps pu être résolu jusqu'au jour où les adversaires se rencontrèrent

53. Voir n. 34 ; cf. le texte attribué à Tномаs Woodsтоск, «The Order of Battle in the Court of Chivalry », dans The Black Book of the Admiralty..., p. 316 et 318, qui renvoie explicitement à la main droite. Henry de BRACton, De legibus et consuetudines angliae, éd. T. Twiss, t. 2, Londres, 1879 , p. 440 (1. 3, tract. 2, ch. 21,2), et le Coutumier bourguignon ne précisent pas de quelle main il s'agit.

54. Sur les «gestes de conciliation» au bas Moyen Âge, cf. N. OfFenstadt, Faire la paix au Moyen Âge. Discours et gestes de paix pendant la guerre de Cent Ans, Paris, 2007. Les recherches sur les émotions ne se concentrent que très rarement sur la dimension «efficace» des gestes «affectifs» (incluant le contact physique). Cf. pourtant E. R. Sмiтн, «An Embodied Account of Self-Other "Overlap" and its Effects", dans E. R. Sмiтн et G. R. Semin éd., Embodied Grounding. Social, Cognitive, Affective, and Neuroscientific Approaches, Cambridge, 2008, p. 148-159 (ici 155), et T. W. Schubert, S. Waldzus et B. SeibT, «The Embodiment of Power and Communalism in Space and Bodily Contact», ibid., p. 160-183 (ici 166-170).

55. Pour l'importance du contexte social, voir M. S. Clark et E. J. Finkel, «Does Expressing Emotion Promote Well-Being? It Depends on Relationship Context», dans L. Z. Tiedens et C. W. Leach éd., The Social Life of Emotions, Cambridge, 2004, p. 105-126, et G. L. Clore et S. Schnall, «Affective Coherence. Affect as Embodied in Attitude, Advertising, and Art», dans E. R. Sмiтн et G. R. Semin éd., Embodied Grounding..., p. 211-236 (ici 221). Pour les gages de bataille-H. NotTARP, Gottesurteilstudien..., p. 276, 289 sq., souligne l'importance de la conciliation et du compromis. Cf. aussi les remarques sur «fere pes» dans Philippe De Beaumanoir, Coutumes du Beauvaisis, éd. A. Salmon, t. 2, Paris, 1900 [réimpr. 1970], p. 433-435, nº 1844-1847 et 1849. Le Coutumier bourguignon, p. 310, souligne la précarité du moment des serments : «[...] cellui qui les oït jurer leur doit defendre que en tenant leurs mains ensemble ils ne se estraignent ne facent mal en aucune maniere.» 
par hasard en présence du roi Charles VII au château de Mehun-sur-Yèvre. Le maréchal de Sévérac aurait ainsi, en sortant de la chambre du roi, littéralement carambolé son adversaire: «... et se rencontrèrent l'un l'autre et heurtèrent des poitrines et s'accolèrent et baisèrent soudainement, pleurant à chaudes larmes, et pardonnèrent l'un à l'autre tous mal-talens, et furent bons amis ensemble ${ }^{56}$ ».

Peu importe que la Chronique de la Pucelle constitue un témoignage bien éloigné des événements : l'argument de la résolution spontanée d'un conflit se retrouve presque à l'identique dans le récit que donne l'historiographe bourguignon Georges Chastellain de la rencontre des adversaires Louis de Luxembourg, comte de Saint-Pol, et Antoine de Crö̈.Après plusieurs années de conflit, le roi Louis XI aurait mis en scène une entrevue à l'insu des protagonistes. Malgré leur résistance initiale lors de cette rencontre inattendue, Louis réussit à prendre et à réunir leurs mains, «au dur et au regret de chacun». Même si les circonstances étaient peu favorables à une réconciliation, la ruse du roi fut couronnée de succès. D'abord les deux acteurs étaient obligés de passer un certain temps dans une situation de «liminalité » dans le sens de Van Gennep: enfermés dans une chambre, ils se trouvaient séparés du reste de la cour. Ensuite, le comte de Saint-Pol et Antoine de Croÿ seraient sortis de celle-ci, «bras à bras comme deux frères », en riant ${ }^{57}$, ce qui équivaut à l'accomplissement d'un rite de passage qui se termine avec l'agrégation des individus en question, c'est-à-dire leur réintégration dans le groupe. Malgré l'absence d'une description détaillée des gestes accomplis, le récit de Chastellain souligne bien le rôle central qu'occupaient les corps des protagonistes dans le déroulement de cette réconciliation.

Ces exemples montrent que les gestes que nous interprétons habituellement dans leur dimension juridique en tant qu'éléments des rituels de conciliation avec une visée avant tout expressive (ils servent donc dans cette perspective à «publier» et confirmer le résultat d'une réconciliation), pouvaient jouer un rôle efficace aux yeux des contemporains. Ainsi, ils dépassent leur statut de signes et entraînent des réactions dont les résultats peuvent être surprenants. Ils deviennent alors de véritables «instruments» de la paix, qui reste quant à elle

56. «Chronique de la Pucelle», dans Chronique de la Pucelle ou Chronique de Cousinot, suivie de la Chronique normande de P. Cochon, éd. A. Vallet de Viriville, Paris, 1859 [réimpr. Genève, 1976], p. 205-339 (ici 236). Sur l'histoire du conflit, voir G. Du Fresne de BeAucourt, Histoire de Charles VII, t. 2, Paris, 1882, p. 126 sq.; C. SAMARAN, La Maison d'Armagnac au XV siècle et les dernières luttes de la féodalité dans le Midi de la France, Paris, 1907 [réimpr. Genève, 1975], p. 100, n. 5. Cf. prochainement K. OsCHEMA, «Falsches Spiel mit wahren Körpern. Freundschaftsgesten und die Politik der Authentizität im franko-burgundischen Spätmittelalter », Historische Zeitschrift, 293 (2011), p. 39-67. Sur la Chronique de la Pucelle, cf. D. FraIoli, «Chronique de la Pucelle», dans G. DunPhy éd., Encyclopedia of the Medieval Chronicle. Vol. I, Leyde, 2010, p. 401 sq.

57. Georges Chastellain, Euvres. 8 vol., éd. J.-B.-M.-C. Kervyn de Lettenhove, Bruxelles 1863-1866 [réimpr. Genève, 1971], vol. IV, p. 131 sq.; cf. K. Oschema, Falsches Spiel... 
un don quasiment divin. En effet, Chastellain observe lui-même qu'il s'agissait d'un «mistère ${ }^{58} »$. Aux yeux de l'historiographe, les événements qu'il décrit relèvent donc d'une influence transcendante. Dans les termes analytiques de nos jours, cette croyance en l'efficacité des gestes se rapproche du domaine des émotions, avec, comme point de référence commun, la mise en œuvre des mécanismes empathiques ${ }^{59}$.

En fait, Chastellain nous fournit d'autres exemples qui rapprochent les phénomènes de la présence et du contact physique avec la disposition affective des personnes concernées. Pour lui, ces effets jouent un rôle lors des rencontres des princes ${ }^{60}$, mais aussi dans le contexte des duels judiciaires. Ainsi, il souligne la réaction affective d'un des protagonistes quand il décrit un duel qui a eu lieu à Valenciennes en 1455 et qui confrontait un certain Jacotin Plouvier avec Mahiénot (ou Matthieu) Cocquiel. Bien que la cérémonie n'ait pas inclus, selon les récits des chroniqueurs ${ }^{61}$, un contact physique entre les combattants, le moment du serment (et donc du contact physique avec l'objet sacré, ici les Évangiles) créa un espace pour la manifestation soit de l'influence divine soit de la réaction affective d'un des adversaires. Car, lorsque Mahiénot embrassa l'objet sacré, il pâlit immédiatement ${ }^{62}$. Du point de vue de la logique narrative, il n'est donc

58. Georges Chastellain, Euvres, vol. IV, p. 132. Pour la notion ambiguë de «mystère», qui peut également signifier une représentation théâtrale, cf. A.-J. GREIMAS et T. MARY KeANe, Dictionnaire du moyen français, Paris, 1992, p. 429.

59. À la différence des mécanismes proposés dans la théorie des «neurones miroir» (mirror neurons), les gestes agissent ici directement à travers les corps des acteurs. Cf. sur les «neurones miroir» N. Zaboura, Das empathische Gehirn. Spiegelneuronen als Grundlage menschlicher Kommunikation, Wiesbaden, 2009, et G. Rizzolatтi, Empathie und Spiegelneuronen. Die biologische Basis des Mitgefuihls, Francfort-sur-le-Main, 2008. Je remercie Rüdiger Schnell (Bâle) d'avoir attiré mon attention sur ces publications!

60. Georges Chastellain, Euvres, vol. IV, p. 7 sq.; cf. P. Contamine, «Les rencontres au sommet dans la France du Xv $v^{e}$ siècle», dans H. Duchrardt et G. Melville éd., Im Spannungsfeld von Recht und Ritual. Soziale Kommunikation in Mittelalter und Früher Neuzeit, Cologne-WeimarVienne, 1997, p. 273-289; G. SCHwEDLER, Herrschertreffen des Spätmittelalters. Formen - Rituale - Wirkungen, Ostfildern, 2008; K. Oschema, Freundschaft..., p. 284-290.

61. Cf. Georges Chastellain, Euvres, vol. III, p. 38-49; Georges Chastellain, Chronique. Les fragments du livre IV, révélés par l'Additional Manuscript 54156 de la British Library, éd. J.-C. Delclos, Genève, 1991, p. 325-327, Olivier de La Marche, Mémoires, éd. H. Beaune et J. D’Arbaumont, t. 2, Paris, 1884, p. 402-407, et Mathieu d'Escouchy, Chronique, éd. G. Du Fresne De Beaucourt, t. 2, Paris, 1863, p. 297-305. Sur ce duel, cf. O. Cartellieri, «Ein Zweikampf in Valenciennes im Jahre $1455 »$, dans W. Keller éd., Probleme der englischen Sprache und Kultur. Festschrift Johannes Hoops, Heidelberg, 1925, p. 169-176; F. MANETTI, «Giudizio di dio a Valenciennes nel 1455 », Publications du Centre européen d'études bourguignonnes, 19 (1978), p. 47-53, et E. LeCUPPRE-Desjardin, La Ville des cérémonies. Essai sur la communication politique dans les anciens Pays-Bas bourguignons, Turnhout, 2004, p. 311-317.

62. Georges Chastellain, Euvres, vol. III, p. 46. 
guère étonnant qu'il ait dû subir ensuite une défaite qui montrait clairement sa culpabilité ${ }^{63}$.

Bien entendu, le récit de Chastellain constitue avant tout une construction littéraire. Or, cette construction joue visiblement sur le registre de l'affectivité humaine dans le contexte qui nous intéresse ici. Cette dimension risque d'être ignorée si on suit une approche exclusivement juridique. Même en se focalisant sur la dimension du rituel, nous devons reconnaître que les effets de ce dernier dépassent la dimension purement expressive d'un moyen de communication symbolique dont la fonctionnalité serait dominée par des effets purement rationnels. Sans vouloir nier l'importance de cet aspect, il me semble nécessaire de réserver une place à la modélisation des émotions ${ }^{64}$. On pourrait ainsi reprendre les observations d'Arlie Hochschild ${ }^{65}$, non pas pour conclure au «manque d'authenticité» dans de nombreuses émotions que nous éprouvons dans notre vie sociale ${ }^{66}$, mais afin de souligner le lien entre les actions extérieures et la disposition affective de l'individu. Dans cette perspective, le corps devient plus qu'un simple moyen de communication (symbolique ou non) et acquiert le statut d'instrument qui sert à façonner les liens sociaux par l'influence qu'il exerce sur l'état affectif des acteurs.

Afin de montrer la plausibilité de cette interprétation, nous pouvons faire mieux qu'énumérer des exemples historiographiques dont l'organisation narrative repose sur cette logique : même si nous regardons du côté des textes juridiques, tels que les lettres de rémission (qui possèdent leurs propres logiques et règles narratives ${ }^{67}$ ), il devient clair qu'à l'époque étudiée, l'exercice de la justice ne

63. Cf. le récit d'un duel qui a eu lieu à Westminster en 1380 chez Thomas Walsingham, Chronica maiora (The St Albans Chronicle). Vol. I: 1376-1394, éd. J. TAYLoR, W. R. Childs et L. WATKIss, Oxford, 2003, p. 356-365 (ici 360): «Armiger autem, quem consciencia stimulabat, nitebatur exceptiones facere, ut quoquo facto sua causa purior effici ualuisset.» (Je souligne.)

64. Cf. P. Ekman et R. J. Davidson, «Affective Science: A Research Agenda», dans Id. éd., The Nature of Emotion. Fundamental Questions, New York, 1993, p. 411-430 (ici 412). Le «façonnement» des émotions au moyen des actions ritualisées est largement négligé par la recherche en faveur de la dichotomie peu fertile entre les actions «rituelles» et «authentiques»; cf. pourtant C. LuTz et G. M. White, «The Anthropology of Emotions », Annual Review of Anthropology, 15 (1986), p. 405436 (ici 413), et T. J. ScheFF, «The Distancing of Emotion in Ritual», Current Anthropology, 18 (1977), p. 483-505.

65. A. HochschiLd, «Emotion Work, Feeling Rules, and Social Structure», American Journal of Sociology, 85 (1979), p. 551-575, et EAD., The Managed Heart. Commercialization of Human Feeling, Los Angeles, 1983.

66. Cf. J.VAn MAAnen et G. Kunda, «"Real Feelings": Emotional Expression and Organizational Culture», dans L. L. Cummings et B. M. Staw éd., Research in Organizational Behaviour, vol. XI, Greenwich, 1989, p. 43-104.

67. Cf. P. Braun, «La valeur documentaire des lettres de rémission», dans La Faute, la répression et le pardon. Actes du $107^{e}$ Congrès national des sociétés savantes (Brest, 1982). Section 
servait pas seulement à établir la vérité et à formuler un jugement adéquat. Premièrement, la grâce du souverain pesait plus lourd que les prescriptions juridiques, de sorte qu'elle créait un espace qui pouvait dispenser les individus en question des conséquences judiciaires de leurs actions. Deuxièmement, et ceci est plus significatif dans notre contexte, l'obtention d'une lettre de rémission présupposait habituellement que le pétitionnaire ait préalablement donné satisfaction à la victime de son crime ou à la famille de celle-ci, «pour éviter "fureur de partie" ${ }^{68} »$. Afin de garantir la concorde et la paix dans la communauté (dans le sens de Tönnies ${ }^{69}$ ), il était donc indispensable de prendre en compte la dynamique des relations entre les individus qui constituent un groupe social ${ }^{70}$. Loin d'être un simple effet de la religion chrétienne et de sa tendance à souligner l'intériorisation de la croyance et la production de l'authenticité religieuse à travers une sorte de mise en parallèle de la «vie interne» de l'âme et des actions externes de l'individu ${ }^{71}$, la production d'une harmonie sociale «authentique» était réalisée dans l'intérêt des communautés et de leurs seigneurs ${ }^{72}$.

L'emploi du contact physique comme instrument d'une pacification obtenue en façonnant des affects faisait partie des stratégies employées dans ce but. Le minuscule détail du «toucher» des adversaires dans la cérémonie qui

de philologie et d'histoire jusqu'à 1610, t. 1, Paris, 1984, p. 207-221 (ici 221). Sur les lettres de rémission en France, voir l'ouvrage fondamental de C. GAUVARD, De grace especial...

68. C. Petit-Dutaillis, Documents nouveaux sur les mœurs populaires et le droit de vengeance dans les Pays-Bas au XV siècle. Lettres de rémission de Philippe le Bon, Paris 1908 (réimpr. Genève, 1975), p. 106. Georges Chastellain, Euvres, vol. III, p. 40 sq., explique que les réfugiés homicides ne pouvaient être admis dans la ville de Valenciennes que si l'homicide qu'ils avaient commis n'était pas «vilain» et qu'ils avaient demandé «francise pour peur des amis et de la justice du prince» (vol. 3, p. 40). Cf. D. Small, The Consumption of Justice. Emotions, Publicity, and Legal Culture in Marseille, 1264-1423, Ithaca (N. Y.), 2003, p. 171 : «In Marseille, however, flight was motivated most often by fear of the family and friends of the victim, not out of fear of capture and incarceration by the sub-viguier.»

69. F. TöNNIES, Gemeinschaft und Gesellschaft. Grundbegriffe der reinen Soziologie, Darmstadt, 1991 [orig. 1887]. Malgré la prise de distance critique dans O. G. OeXLE, «"Das Mittelalter" - Bilder gedeuteter Geschichte» et dans J. M. BAK et al. éd., Gebrauch und Missbrauch des Mittelalters, 19.-21. Jahrhundert/Uses and Abuses of the Middle Ages: 19th-21st Century/Usages et mésusages du Moyen Âge du XIX au XXI siècle, Munich, 2009, p. 21-43 et 345-349 (ici 33), les catégories de la «communauté» (Gemeinschaft) et de la «société » (Gesellschaft) me semblent bien choisies pour saisir les particularités des cultures pré-modernes dans un sens «idéaltypique».

70. Cf. dans le contexte des lettres de rémission P. TexieR, «La rémission au XIV siècle: significations et fonctions », dans La Faute..., p. 193-205, (ici 197-199).

71. Cf. par ex. les contributions dans K. Philipowski et A. Prior éd., Anima und sêle. Darstellungen und Systematisierungen von Seele im Mittelalter, Berlin, 2006.

72. C. Petit-Dutaillis, Documents nouveaux..., p. 105 sq. Ceci n'empêche pas les tentatives du pouvoir central de s'imposer dans la mesure du possible, souvent au détriment des coutumes locales, cf. ibid., p. 108-112. 
précède le combat n'étant guère explicable par une approche exclusivement juridique, il devient cependant compréhensible quand on y inclut la perspective des contemporains: pour eux, le contact avec un autre individu, même s'il s'agissait d'un adversaire, pouvait entraîner des conséquences bénéfiques qui allaient éventuellement jusqu'à la résolution spontanée d'un conflit. Dans une culture qui développait une attitude négative envers les gages de bataille en tant qu'instrument juridique ${ }^{73}$, mais qui restait cependant réticente à accepter l'abolition de cette tradition, il devait être évident qu'il fallait faire tous les efforts possibles afin de permettre la réalisation d'un «mystère».

Si l'on accepte cette perspective, le contact physique des adversaires devient un indice révélateur du rôle que les sociétés du bas Moyen Âge accordaient aux effets des émotions. L'interprétation de cet acte presque imperceptible ouvre des pistes qui nous mènent à l'analyse de ce qu'on pourrait appeler des systèmes de croyance, des stratégies du emotional labour et des régimes affectifs des cultures en question ${ }^{74}$. Le fait que je n'aie pu identifier jusqu'à présent aucun exemple concret qui témoigne du succès de cette pratique invite évidemment à douter, mais n'invalide aucunement l'existence de la stratégie observée ${ }^{75}$. Sur le plan théorique, toute une série de sources exprime le souci de permettre une pacification avant le combat ${ }^{76}$.

Cela ne veut pourtant pas dire que chacun de ces complexes est toujours gouverné par une logique cohérente et conséquente. En fait, l'exemple de Chastellain et du récit qu'il donne du duel de Valenciennes montre bien les difficultés auxquelles pouvaient se heurter les contemporains: si la réaction

73. Voir Olivier de La Marche, «Livre... », p. 3 sq. et 28 ; cf. Henry de Bracton, De legibus..., t. 4, Londres, 1881, p. 368 (1. 4, tract. 5, ch. 1, 1): «Sed cum restringendæ sunt lites potius quam laxandæ, ut vitentur duella \& magnæ assisæ $[\ldots] »$.

74. Cf. B. H. Rosenwein, Emotional Communities in the Early Middle Ages, Ithaca (N. Y.), 2006. Il me semble qu'une approche qui combine la «micro-histoire» des gestes avec leur interprétation au carrefour des textes normatifs et descriptifs peut servir à éviter les failles de la «macro-théorie» $\mathrm{du}$ «Zivilisationsproze $\beta$ » sur la base des concepts heuristiques parfois peu aptes à saisir les particularités des systèmes épistémiques des cultures en question, cf. par exemple J. R. MAJoR, " "Bastard Feudalism" and the Kiss: Changing Social Mores in Late Medieval and Early Modern France», The Journal of Interdisciplinary History, 17 (1986-87), p. 509-535. On regrettera donc que D. Small, Consumption of Justice..., p. 160-206, se concentre uniquement sur les gestes de la punition et la «coercive force » exercée par la punition du corps.

75. Je remercie Klaus Krüger (Halle) pour sa remarque pertinente: l'existence d'une bataille «évitée» au moyen de la pratique esquissée fournirait un excellent argument pour mon analyse. La brève description d'un duel dans les «Annales prioratus de Wigornia», dans Annales monastici, éd. H. R. LuARD, vol. IV, Londres, 1869, p. 467-468 (pour l'année 1275), n'est pas assez détaillée: «Septimo kal. Julii fuit duellum in pastura de Hardewik pro ecclesia de Temedebyre [i.e. Tenbury], sed facte pace remansit ecclesia cum abbate de Lyre [i.e. Ralph II].»

76. Cf. n. 55 et le Coutumier bourguignon, p. 22: «Se composicion de bataille soit faite devant le cop ou aprés le cop, je [i.e. le duc de Bourgogne] en auray XXXII s. et V d.» 
de Mahiénot, qui pâlit lors de son serment, trahit indubitablement sa position défectueuse ${ }^{77}$, il ne saisit pourtant pas l'occasion pour se confesser et éviter ainsi la cruauté du combat que les historiographes aristocratiques décrivent avec beaucoup de dégoût ${ }^{78}$. Or, du point de vue des spectateurs, il s'agissait ici d'un défaut individuel qui ne les incitait pas nécessairement à douter du cadre général de la pratique judiciaire en question ou de l'efficacité des gestes qu'il contenait ${ }^{79}$.

Klaus Oschema - Historisches Seminar der Universität Heidelberg, Grabengasse 3-5, 69117 Heidelberg, Allemagne

77. Cf. n. 62.

78. Cf. Georges Chastellain, Chronique, éd. Delclos, p. 325, sur la réaction de Philippe le Bon: «Si en prist pitié au duc, car estoit la plus inhumaine chose qu'onc homme veist.» Le récit continue avec les détails du carnage; la «victime» est comparée à un animal de boucherie (ibid., p. 325-327). Cf. Olivier de LA MARChe, Mémoires, vol. II, p. 407, avec des raisonnements qui relèvent plutôt du registre de l'esthétique et de la morale nobiliaire que de la compassion. MATHIEU D’Escouchy, Chronique, vol. II, p. 304 sq., critique surtout la cruauté de Jacotin.

79. Je souligne que ma présentation se concentre délibérément sur la dimension des gestes de proximité employés dans ce rituel; or, il est évident que ce dernier incluait également l'utilisation d'objets sacrés. En outre, les croyances des contemporains soulignaient la présence divine dans le moment de combat. Si cet imaginaire fut certes essentiel pour la perception du duel judiciaire à l'époque, il me semble qu'il n'exclut pas la possibilité de traduire les mécanismes sociaux autour du geste qui m'a intéressé ici dans la langue analytique des «émotions». 


\section{Toucher et être touché : gestes de conciliation et émotions dans les duels judiciaires}

Afin de compléter les discussions théoriques concernant les paramètres d'une histoire des émotions, la présente contribution analyse un détail du cérémoniel des «gages de bataille» bas-médiévaux qui ne peut pas être expliqué de manière satisfaisante au moyen des approches classiques de l'histoire du droit ou de la «communication symbolique». Une stratégie analytique qui inclut la dimension affective des actions ritualisées montre que le geste effectué par les adversaires, qui se tiennent par la main lors du dernier des serments qu'ils prononcent avant la bataille, s'harmonise bien avec les tendances contemporaines à éviter, dans la mesure du possible, la violence du combat. Dans cette perspective, les adversaires exécutent un geste de pacification (ou de réconciliation) potentiel qui pourrait les amener à ne pas en venir à la confrontation armée: de manière analogue, les historiographes de l'époque se réfèrent à plusieurs reprises au motif de la réconciliation spontanée qui résulte d'un contact physique, même accidentel. Même si le succès réel de cette pratique semble avoir été plutôt restreint, l'organisation rituelle visait à modifier la disposition affective des acteurs par le biais de moyens dont l'efficacité potentielle a pu être montrée par la recherche en psychologie sociale moderne.

Gage de bataille - émotion - gestes - rituel - France - XIV et XV siècles.

\section{To touch and to be touched : gestures of conciliation and emotions in trial by combat}

In order to complement the current theoretical debates on the parameters of a «History of Emotions », the present contribution analyses a ceremonial detail of late medieval trials by combat which cannot be explained satisfactorily within the classical approaches of Legal History or «symbolic communication». An analytical strategy that includes the affective dimension of ritualised action shows that the gesture executed by the adversaries, who held each other by the hand during the last sermon they had to swear before their battle, coincided with contemporary tendencies to avoid, if possible, the violence of combat. In that sense, the adversaries were forced to perform a potential gesture of pacification (or reconciliation) which could prompt them to avoid armed confrontation. This can be compared to a motif that the historiographers of the period refer to repeatedly, i.e. the idea of spontaneous reconciliation resulting from (even accidental) physical contact. Although the real success of such a practice seems to have been quite limited, the organisation of the ritual intended to modify the actors'affective state by means that have been shown to be potentially efficient in modern socio-psychological research.

Trial by combat - emotions - gestures - ritual - France - 14th and 15th centuries. 
